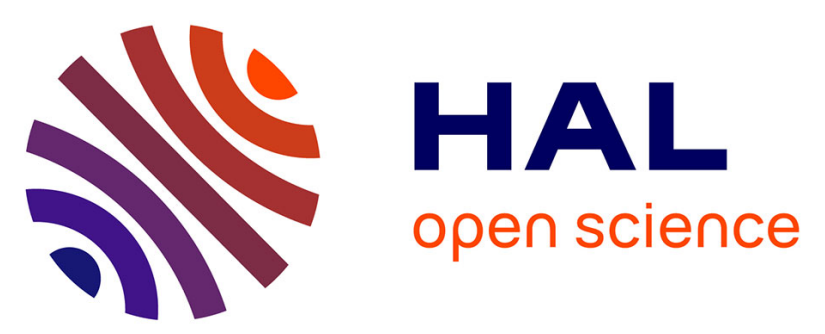

\title{
Les origines du culte des saints martyrs Fuscien, Victoric et Gentien d'après les sources manuscrites
}

\author{
Dominique Paris-Poulain
}

\section{To cite this version:}

Dominique Paris-Poulain. Les origines du culte des saints martyrs Fuscien, Victoric et Gentien d'après les sources manuscrites. Revue d'histoire de l'Eglise de France, 2019, 105, pp.235 - 251. 10.1484/j.rhef.5.120353 . halshs-03389766

\section{HAL Id: halshs-03389766 \\ https://shs.hal.science/halshs-03389766}

Submitted on 21 Oct 2021

HAL is a multi-disciplinary open access archive for the deposit and dissemination of scientific research documents, whether they are published or not. The documents may come from teaching and research institutions in France or abroad, or from public or private research centers.
L'archive ouverte pluridisciplinaire HAL, est destinée au dépôt et à la diffusion de documents scientifiques de niveau recherche, publiés ou non, émanant des établissements d'enseignement et de recherche français ou étrangers, des laboratoires publics ou privés. 


\section{LES ORIGINES DU CULTE DES SAINTS MARTYRS FUSCIEN, VICTORIC ET GENTIEN D'APRÈS LES SOURCES MANUSCRITES}

Durant la période médiévale, les trois saints martyrs Fuscien, Victoric et Gentien furent l'objet d'une vénération toute particulière dans l'Église d'Amiens. Cette dévotion se concrétisa, entre autres, par la fondation en 1105, sur les lieux présumés de leur martyre, d'une abbaye placée sous le patronage de saint Fuscien ${ }^{1}$, et par la réalisation, dans les années 1225-1230, dans l'église paroissiale de Sains (aujourd'hui Sains-en-Amiénois), d'un exceptionnel monument sculpté à l'endroit même où, d'après la tradition, avaient été inhumés leurs restes corporels ${ }^{2}$. Des investigations menées en 1663 sous le monument gothique ont permis de mettre au jour un sarcophage contenant trois capsulae servant de réceptacles à quelques ossements ${ }^{3}$. Cette découverte semble accréditer la dispersion des reliques de ces saints, que plusieurs sources signalent dès le $\mathrm{Ix}^{\mathrm{e}}$ siècle. Mais, au regard du témoignage concordant des plus anciennes transcriptions conservées de la recension gallicane du martyrologe hiéronymien et du récit hagiographique qui leur fut consacré, il ne fait aucun doute que leur culte était déjà bien en place au viII ${ }^{\mathrm{e}}$ siècle.

L'objectif de cette étude est d'interroger ces témoins manuscrits afin de tenter de préciser les circonstances dans lesquelles le culte de ces martyrs a pu voir le jour. À quel moment leurs noms ont-ils été inscrits dans le martyrologe? Dans quel contexte fut rédigée la version primitive du récit hagiographique? Quels en furent les promoteurs potentiels? Autant de questions auxquelles les réponses apportées, en raison du déficit des sources,

1. Un vidimus de la charte de fondation est conservé aux arch. dép. de la Somme sous la cote

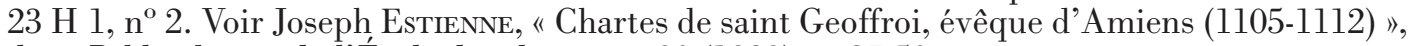
dans Bibliothèque de l'École des chartes, t. 90 (1929), p. 37-50.

2. En l'attente de la publication de mes recherches en cours sur ce monument, voir abbé Henri Bouvier, "Le tombeau des saints Fuscien, Victoric et Gentien et l'épitaphe mérovingienne de l'église de Sains », dans Bulletin de la Société des Antiquaires de Picardie, t. 26 (1913-1914), p. 19-43.

3. Amiens, bibl. mun., ms. 831 E (Jean Pagès, Notices historiques sur la ville d'Amiens, $12^{\mathrm{e}}$ dialogue, 1718), p. 66. Voir Manuscrits de Pagès, marchand d'Amiens, écrits à la fin du $17^{e}$ et au commencement du $18^{e}$ siècle sur Amiens et la Picardie, éd. Louis Douchet, t. IV, Amiens, 1860, p. 343-344. 
ne pourront qu'être conjecturales, mais que l'on ne doit pas pour autant s'interdire de poser.

\section{Victoric et Fuscien dans les versions gallicanes du martyrologe hiéronymien}

Les noms de Victoric et Fuscien, dans cet ordre et à l'exclusion de celui de Gentien, apparaissent dans les recensions les plus anciennement conservées de la version gallicane du martyrologe hiéronymien, parmi les saints commémorés le 11 décembre (3 des ides de décembre). L'éloge précise le lieu de leur martyre sur le territoire des Ambiani. La formulation utilisée dans les manuscrits offre des variations plus ou moins accentuées, résultant des différentes transcriptions qui se sont succédé lors de la transmission de l'ouvrage. Ainsi, dans les codices pleniores, que l'on pense, depuis les travaux de Louis Duchesne ${ }^{4}$, être les chefs de file de deux lignes de transmission de la tradition manuscrite du martyrologe, l'écart se limite à une voyelle dans la graphie du premier nom : "In Gall[iis] Ambianis Victorici et Fusciani mar[tyrum]» dans le codex Epternacensis ${ }^{5}$, "In Gall[iis] Ambianis Victurici et Fusciani mar[tyrum] ", dans le codex Wissenburgensis ${ }^{6}$. Dans la version plus ou moins abrégée du codex Corbeiensis brevius, la précision topographique, formulée différemment, évoque tout autant l'espace géographique de l'antique civitas des Ambiani dont hérita le diocèse d'Amiens que la cité épiscopale stricto sensu : "Ambianis civit[as] nat[alis] Victurici et Fusciani mar[tyrum] " 7 . Quant aux autres versions abrégées du martyrologe hiéronymien, livrées par les sacramentaires d'Autun et de Gellone, on notera la confusion commise dans le codex Gellonense entre Fuscianus et Faustiani, et l'absence de la mention spécifiant le statut de martyr, qui est cependant la règle adoptée dans cet ouvrage : "Ambianus Victuri[ci] Fusciani m[a]r[tyrum]" dans le sacramentarium Augustodunense ${ }^{8}$, "Ambianis Victurici Faustiani ",dans le sacramentarium Gellonense ${ }^{9}$.

La date et le lieu de copie de chacun de ces manuscrits ainsi que leurs destinations respectives ont été largement débattues. Je me contenterai de rappeler ce que nous pouvons considérer comme acquis. Le martyrologe

4. Martyrologium Hieronymianum, éd. Giovanni Battista De Rossi et Louis Duahesne, Acta sanctorum, Novembris, t. II, part. I, Bruxelles, 1894, p. viII-XXI, et Hippolyte Delehaye et dom Henri Quentin, Acta sanctorum Novembris, t. II, pars posterior, Bruxelles, 1931.

5. Bibl. nat. de France, Lat. 10837, fol. 3r. Voir Elias A. Lowe, Codices Latini Antiquiores : A Paleographical Guide to Latin Manuscripts prior to the Ninth Century, t. V, France, Paris, Oxford, 1950, n⿳0 605.

6. Wolfenbüttel, Herzog-August-Bibliothek, Cod. Guelf. 81 Weiss, fol. 99r. Voir E. A. LowE, Codices Latini Antiquiores..., t. IX, Germany, Maria Laach-Würzburg, Oxford, 1959, n 1393.

7. Bibl. nat. de France, Lat. 12260, fol. 11v. Voir E. A. Lowe, Codices Latini Antiquiores..., t. $\mathrm{V}, \mathrm{n}^{\mathrm{o}} 641$.

8. Berlin, Staatsbibliothek Preussischer Kulturbesitz, ms. Phillipps 1667, fol. 199v. Voir E. A. Lowe, Codices Latini Antiquiores..., t. VIII, Germany, Altenburg-Leipzig, Oxford, 1959, $\mathrm{n}^{\mathrm{o}} 1056$.

9. Bibl. nat. de France, Lat. 12048, fol. 274v. Voir E. A. Lowe, Codices Latini Antiquiores..., t. $\mathrm{V}, \mathrm{n}^{\mathrm{o}} 618$. 
d'Echternach fut copié par un scribe du nom de Laurentius, de formation anglo-saxonne, pour le monastère fondé en 698 par le Northumbrien Willibrord. Le texte fut réuni à un calendrier liturgique, daté entre 702 et 706, auquel le scribe emprunta certaines entrées. On peut sans trop de marge d'erreur dater la transcription de cette version dans les premières années du VIII ${ }^{\mathrm{e}}$ siècle et la considérer comme le plus ancien témoin conservé de la recensio Gallica. Le scriptorium dont le manuscrit est issu n'a cependant pu être localisé avec certitude; les différentes hypothèses oscillent entre l'Irlande, la Northumbrie et Echternach ${ }^{10}$. L'exemplaire de Wissembourg fut, quant à lui, copié au plus tard en 772, soit à l'abbaye Saint-Wandrille de Fontenelle, comme on le suppose habituellement, soit, comme l'ont soutenu Jean Laporte et Felice Lifshitz, à et pour l'église Saint-Servais de Maastricht, avant de rejoindre l'abbaye Saint-Pierre de Wissembourg ${ }^{11}$. Il n'est pas sans intérêt de souligner que l'Église d'Amiens, à l'exception de la mention relative à Victoric et Fuscien, est quasiment terra incognita pour les rédacteurs de ces deux manuscrits. L'écriture de «type Maurdramne» observée dans l'exemplaire de Corbie invite à placer la réalisation de celui-ci dans le dernier quart du viII ${ }^{\mathrm{e}}$ siècle ${ }^{12}$. Cette fourchette chronologique pourrait être resserrée si l'on accepte la suggestion convaincante de Felice Lifshiz d'attribuer la commande du codex, dans lequel le martyrologe précède la Regula pastoralis de Grégoire le Grand, à Adalhard peu après 781, alors qu'il cumulait, en plus de ses responsabilités à la tête de l'abbaye, les fonctions de conseiller de Charlemagne et de régent du jeune roi d'Italie, Pépin ${ }^{13}$. Quant aux deux

10. Pour l'identité du scribe et le scriptorium voir en dernier lieu Dáibhí Ó Grórnín, «Rath Melsigi, Willibrord and the Earliest Echternach Manuscripts ", dans Peritia, t. 3 (1984), p. 17-42 ; Rosamond MaKitterick, «The Diffusion of Insular Culture in Neustria between 650 and 850 : The Implications of the Manuscript Evidence », dans Hartmut Atsma (éd.), La Neustrie : les pays au nord de la Loire de 650 à 850 (colloque historique international), Sigmaringen, 1989 (Beihefte der Francia, 16), vol. II, p. 395-432, spéc. p. 426, et R. MaKitTERIaK, " Le scriptorium d'Echternach aux huitième et neuvième siècles ", dans Michel PoLfER (éd.), L'évangélisation des régions entre Meuse et Moselle et la fondation de l'abbaye d'Echternach ( $V^{e}-I X^{e}$ siècle) : actes des 10 ${ }^{\text {es }}$ Journées lotharingiennes, 28-30 octobre 1998, Centre universitaire de Luxembourg, Luxembourg, 2000 (Publications de la section historique de l'Institut grand-ducal de Luxembourg, 117. Publications du CLUDEM, 16), p. 499-522.

11. Dom Jean Laporte, "Les recensions de Fontenelle du martyrologe hiéronymien et l'histoire du monastère ", dans Revue Mabillon, t. 29 (1939), p. 1-16, et Felice Lifshitz, The Name of the Saint : The Martyrology of Jerome and Access to the Sacred in Francia (627-827), Notre Dame (Ind.), 2006 (Publications in Medieval Studies), p. 44-56.

12. Terence A. M. Bishop, "The Script of Corbie : A Criterion », dans Varia Codicologica : Essays Presented to G. I. Lieftinck, t. I, Amsterdam, 1972 (Litterae textuales), p. 9-16, spéc. p. 10 et n. 7 .

13. David Ganz, Corbie in the Carolingian Renaissance, Sigmaringen, 1990 (Beihefte der Francia, 20), p. 134-135, et F. Lifshitz, The Name of the Saint ..., p. 82-89. Le martyrologe comporte quelques additions de la fin du $\mathrm{Ix}^{\mathrm{e}}$ siècle, parmi lesquelles figure le nom de Gentianus. Contrairement à ce qu'affirme Michèle Gaillard, ni la translation de Gentien ni l'invention des trois martyrs ne font partie des célébrations inscrites dans ce martyrologe (Michèle GaILLARD, "Un "cycle" hagiographique du haut Moyen Âge en Gaule septentrionale : les Passions des martyrs de Riciovar", dans Hagiographica, t. 21 (2014), p. 1-28, spéc. p. 1, n. 2). Cette inexactitude provient d'une confusion avec le manuscrit Lat. 12410 de la Bibl. nat. de France (voir la n. 25 ci-dessous). Un indice permettrait de préciser davantage encore la date de réalisation du manuscrit Lat. 12260 si l'on parvenait à identifier le diacre Odilardus, dont l'obit a été ajouté à la fin du texte, au fol. 12, par le scribe responsable de cette recension. 
sacramentaires gélasiens d'Autun ${ }^{14}$ et de Gellone ${ }^{15}$, ils ont été composés un peu plus tardivement au cours de la dernière décennie du vinI siècle, le premier pour la cathédrale d'Autun, le second peut-être au monastère SainteCroix (plus tard Saint-Faron) de Meaux et pour la cathédrale de Cambrai sous l'épiscopat d'Hildoard (vers 790-816), avant d'être transféré à Gellone. Mais le martyrologe abrégé de ce volume fut probablement copié et ajouté au sacramentaire dans ce monastère peu après sa fondation en $804{ }^{16}$.

L'inventaire des martyrologes hiéronymiens du viII ${ }^{\mathrm{e}}$ siècle mentionnant nos deux saints martyrs amiénois s'arrête là. Les autres exemplaires contemporains de ceux-ci, au nombre de deux, ayant pu leur consacrer un éloge, nous sont malheureusement parvenus amputés des derniers mois de l'année ${ }^{17}$.

Sachant que toutes ces versions dérivent d'un fond commun plus ou moins amplifié, mis en chantier en Gaule aux alentours de 600, on ne peut écarter l'hypothèse que les noms de nos deux saints martyrs aient été inscrits dans l'une des toutes premières compilations. On s'accorde généralement à relier les prémisses de leur culte à la recension rédigée à Auxerre sous l'épiscopat d'Aunaire, entre 592 et $603{ }^{18}$. Mais l'absence de preuve irréfutable en faveur d'une entrée aussi précoce dans la version gallicane laisse le champ libre aux conjectures et à l'éventualité que les noms de Victoricus et Fuscianus aient pu apparaitre pour la première fois dans des compilations ultérieures, à l'exemple de Colomban, mort en 615 et mentionné dans tous les Codices pleniores. Lorsque les informations biographiques fiables nous échappent, il est presque toujours impossible de savoir à quel moment précis un nom a pu être introduit dans le texte de référence. En revanche, l'indication topographique, mentionnée dans la majorité des cas, offre la possibilité de déterminer le lieu où cet ajout a pu avoir été effectué. Ainsi, la manière dont la recension gallicane s'est constituée, par adjonctions successives au gré de la propagation du texte à travers la Gaule et en fonction des intérêts des clercs et des communautés qui en avaient l'usage, laisse à penser que le copiste responsable de l'insertion du nom des deux martyrs amiénois appartenait à un scriptorium situé dans le

La mémoire du diacre, dont aucun autre document corbéien ne fait état, est rappelée le 13 octobre.

14. Liber sacramentorum Augustodunensis, éd. Odilo Heiming, Turnhout, 1984 (Corpus Christianorum. Series Latina, 159 B).

15. Liber sacramentorum Gellonensis, éd. Antoine Dumas et Jean Deshusses, Turnhout, 1981 (Corpus Christianorum, Series Latina, 159 et 159 A).

16. Arno Bonst, Die karolingische Kalenderreform, Hanovre, 1998 (Monumenta Germaniae historica. Schriften, 46), p. 224-225.

17. Le plus ancien des deux manuscrits, un martyrologe-calendrier (Bibl. nat. de France, Lat. 14086, fol. 4r-5v), a été composé au monastère de Moutiers-Saint-Jean, dans le diocèse de Langres, soit entre la fin du viI ${ }^{\mathrm{e}}$ siècle et le début du viII ${ }^{\mathrm{e}}$ siècle si l'on suit Pierre SALmon, «Le martyrologe-calendrier conservé dans le ms. L. 14086 de Paris et ses origines ", dans Revue bénédictine, t. 56 (1945-1946), p. 42-57, soit au cours de la première moitié du viII siècle, comme le pense E. A. Lowe, Codices Latini Antiquiores..., t. V, $\mathrm{n}^{\circ}$ 664. Le second, le codex Bernensis (Berne, Burgerbibliothek, Cod. 289, fol. 52v-129v ; voir E. A. Lowe, Codices Latini Antiquiores..., t. VII, Switzerland, Oxford, 1956, nº 861) fut copié après 766 pour Saint-Avold de Metz.

18. Dom Jacques Dưsois, Les martyrologes du Moyen Age latin, Turnhout, 1978 (Typologie des sources du Moyen Âge occidental, 26), p. 33. 
diocèse d'Amiens, soit au sein même de la cité épiscopale, soit dans un monastère proche.

Compte tenu du caractère lacunaire de la documentation, l'activité d'un atelier amiénois n'est pas assurée avant les premières années du Ix ${ }^{\mathrm{e}}$ siècle ${ }^{19}$. Mais, ainsi que l'a pertinemment remarqué Émile Lesne, un évêque pouvait également commander aux ateliers des monastères faisant partie de son episcopium les livres dont il avait besoin ou qui étaient nécessaires à la célébration liturgique ${ }^{20}$.

Par ses origines, son rayonnement et l'importance de son atelier, l'abbaye de Corbie offrait toutes les conditions pour satisfaire les sollicitations de l'évêque d'Amiens : en témoigne la Chronica universalis Alexandrina Latina rédigée par l'évêque Georgius (769-798), qui fut précisément copiée à Corbie ${ }^{21}$. Fondée à l'initiative de la reine Bathilde entre 657 et $661^{22}$, l'abbaye accueillit une première communauté composée de moines venus de Luxeuil qui, sous la direction de Theodefridus, contribuèrent à créer ce qui deviendra très rapidement l'un des scriptoria les plus actifs du Nord de la Gaule. La probabilité qu'un exemplaire du martyrologe hiéronymien ait pu faire partie des ouvrages que les moines apportèrent de leur établissement d'origine est parfaitement envisageable. Les plus anciens manuscrits de la bibliothèque de Corbie furent soit produits à Luxeuil soit écrits dans la nouvelle fondation par d'anciens moines de Luxeuil ${ }^{23}$. Toutefois, et en dépit du fait que le monastère luxovien eut très probablement un rôle important dans la transmission de la recensio Gallica $^{24}$, il ne semble pas concevable que le modèle utilisé par les moines responsables du martyrologe copié à Corbie du temps où Adahalard y séjournait provienne d'une main luxovienne, car, dans le manuscrit Latin 12260 de la Bibliothèque nationale de France, les noms des figures majeures de l'abbaye de Luxeuil, tels Colomban (au 9 des calendes de décembre), son fondateur, ou Eustasius (au 3 des nones d'avril), le successeur de ce dernier n'ont pas été

19. Elias A. Lowe a rapproché plusieurs manuscrits qui révèlent la présence d'un scriptorium dans la cité épiscopale autour de l'an 800 (E. A. LowE, Codices Latini Antiquiores..., t. VI, France, Abbeville-Valenciennes, Oxford, 1953, $\mathrm{n}^{\circ}$ 821). Cette proposition est reprise par Bernhard Bischoff, Manuscripts and Libraries in the Age of Charlemagne, trad. angl., Cambridge, 1994 (Cambridge Studies in Palaeography and Codicology, 1), p. 25.

20. Émile Lesne, Histoire de la propriété ecclésiastique en France, t. IV Les livres, "scriptoria » et bibliothèques du commencement du VIII è la fin du XI siècle, Lille, 1938 (Mémoires et travaux publiés par les professeurs des facultés catholiques de Lille, 46), p. 92.

21. Bibl. nat. de France, Lat. 4884. Voir E. A. Lowe, Codices Latini Antiquiores..., t. V, $\mathrm{n}^{\mathrm{o}} 560$.

22. La date de fondation est incertaine : l'original du diplôme de fondation est perdu, et seules nous en restent des copies dont la plus ancienne figure dans le cartulaire de Corbie daté du troisième quart du Ix ${ }^{\mathrm{e}}$ siècle (Berlin, Deutsche Staatsbibliothek, Phillipps 1776, fol. 126v-128r). En dernier lieu, voir Josiane BARBier et Laurent Morelle, « Le diplôme de fondation de l'abbaye de Corbie (657/661) : contexte, enjeux et modalités d'une falsification », dans Revue du Nord, t. 93 (2011), p. 613-654. Pour les débuts de l'histoire de l'abbaye, voir également D. Ganz, Corbie in the Carolingian Renaissance..., p. 14-35.

23. Ibid., p. 36-40 et 124-126.

24. Sur la rédaction primitive de la recensio Gallica et le débat contradictoire autour de son origine auxerroise ou luxovienne, voir F. Lifshitz, The Name of the Saint ..., p. 13-19, et Pádraig Ó Riain, Feastdays of the Saints : A History of Irish Martyrologies, Bruxelles, 2006 (Subsidia hagiographica, 86), p. 2-7. 
mentionnés. On constate en revanche que les moines de Corbie ont produit un exemplaire de la recension gallicane adapté à l'usage de leur propre abbaye. Ils ont introduit les entrées de Baldechildis (au 3 des calendes de février), de Teodefridus (au 7 des ides d'octobre), et celles qui sont relatives à la dédicace de trois des églises du monastère, consacrées respectivement à saint Jean l'Évangéliste (au 3 des calendes de février), à saint Pierre (au 5 des calendes d'août) et aux saints Albin et Marcellin (au 11 des calendes d'octobre) ${ }^{25}$. À ces nouvelles entrées se sont jointes celles qui se rapportent aux saints les plus éminents du diocèse: Firmin (Firminus, au 7 des calendes d'octobre), Riquier (Richarius, au 6 des calendes de mai) et Valery (Walaricus, au 4 des nones d'avril), complétées par celle de Lucien (Lucianus, au 6 des ides de janvier), premier évêque supposé du diocèse voisin de Beauvais. Ces mentions propres à l'abbaye de Corbie, et plus largement au diocèse d'Amiens, apparaissent pour la première fois dans le manuscrit Latin 12260, mais, étant donné la date de sa réalisation, il ne fut sans doute pas le premier exemplaire enrichi de ces ajouts ${ }^{26}$.

À l'évidence, les moines de Corbie eurent à cœur d'actualiser la liste des noms de saints en y ajoutant ceux qui, en vertu de leur proximité, pouvaient exercer un pouvoir de protection efficace. La décision prise, bien avant, d'inscrire dans la recensio Gallica les noms de Victoricus et Fuscianus relevait des mêmes préoccupations. L'initiative pourrait revenir à Theodefridus, promu, d'après plusieurs sources, évêque d'Amiens probablement dès $673^{27}$, mais l'intervention éventuelle de Bertefredus (644-vers 670), son prédécesseur à la tête de l'évêché, n'est pas non plus à négliger. Formé ou influencé par le monachisme luxovien, Bertefredus a noué d'étroites relations avec l'abbaye de Corbie, en lui concédant un privilège épiscopal ${ }^{28}$

25. L'omission de l'église dédiée à saint Étienne s'explique difficilement. Mais la mémoire du protomartyr est rappelée le jour où est célébrée l'invention de ses reliques (au 3 des nones d'août).

26. Cette version a servi de modèle à deux recensions copiées à Corbie par Névelon au début du xiI ${ }^{\mathrm{e}}$ siècle (Bibl. nat. de France, Lat. 17767, fol. 193r-196r, et Lat. 12410, fol. 4r-15r ; voir Christian DE MÉRINDOL, «Le modèle du recueil de Névelon : nouvel aperçu sur l'abbaye de Corbie au x siècle », dans Dominique Poulain et Michel Perrin (éd.), L'art du haut Moyen Âge dans le Nord-Ouest de la France : actes du colloque de St-Riquier (22-24 sept. 1987), Greifswald, 1993 (Wodan, 23. Serie 3, Tagungsbände und Sammelschriften, 10. Greifswalder Beiträge zum Mittelalter, 8), p. 277-325.

27. La date de l'élévation et l'identification du siège épiscopal ont fait l'objet de discussions. Sur la foi d'un diplôme de Thierry III, daté d'entre 673 et 690, et d'une charte de l'évêque du Mans Aiglibertus, datée de 683, à laquelle Theodefridus a souscrit en tant qu'évêque, on peut placer l'épiscopat de ce dernier entre 673 et 683. Le diplôme royal fait état du rôle important qu'il a joué dans l'élection de ses deux premiers successeurs à Corbie, ce qui permet d'identifier son siège épiscopal à Amiens avec une quasi-certitude. Le témoignage de la première rédaction de la Vita Bathildis va dans le même sens (Vita sanctae Balthildis, éd. Bruno Krusch, MGH, SS rer. Merov., t. II, Hanovre, 1888, p. 490 ; voir Léon Levillain, Examen critique des chartes mérovingiennes et carolingiennes de l'abbaye de Corbie, Paris, 1902 (Mémoires et documents publiés par la Société de l'École des chartes, 5), p. 59-68 et 232-234, et D. Ganz, Corbie in the Carolingian Renaissance..., p. 18).

28. Pour la traduction en français du texte de la charte, datée du 6 septembre 664, et son analyse, voir L. Morelle, "Le statut d'un grand monastère franc : Corbie (664-1050) », dans François Bougard (éd.), Le christianisme en Occident, du début VII au milieu XI siècle, Paris, 1997 (Regards sur l'histoire, 117. Histoire médiévale), p. 203-224, spéc. p. 205-215. 
et en y trouvant un lieu de retraite conforme à ses aspirations durant le carême ${ }^{29}$.

L'hypothèse qu'une compilation de la recensio Gallica adaptée à l'église d'Amiens ait pu voir le jour à Corbie, une fois la communauté définitivement installée et alors que la nécessité de se fournir en instruments de culte se faisait sentir, est plus que vraisemblable. Selon cette conjecture, la version transcrite à Corbie, peu après la fondation de l'abbaye, précéderait de facto la réalisation du martyrologe d'Echternach.

Cela soulève la question de la diffusion de l'exemplaire corbéien enrichi des noms de Fuscien et de Victoric. La transmission a pu s'effectuer par différentes voies. L'activité croissante du scriptorium de l'abbaye picarde a fourni un certain nombre d'ouvrages qui ont alimenté les bibliothèques d'autres établissements. De surcroît, l'adoption de la règle de saint Benoît ${ }^{30}$ imposait à l'abbaye un devoir d'hospitalité qu'elle dût accomplir régulièrement en raison de sa situation géographique privilégiée, à proximité d'une ville située au carrefour des principaux axes routiers qui irriguaient le Nord de la Gaule ${ }^{31}$. L'abbaye constituait une étape idéale, notamment pour les voyageurs anglosaxons qui, sur le chemin de Rome, empruntaient la via rectissima reliant Amiens au port de Quentovic ${ }^{32}$. L'opportunité d'informer leurs hôtes de passage, curieux de connaître les noms de saints vénérés dans le diocèse et dont la mémoire était digne d'être honorée, s'est certainement présentée à plus d'une occasion aux responsables du monastère.

29. Voir Jean Heuclin, Hommes de Dieu et fonctionnaires du roi en Gaule du Nord du Ve au IX ${ }^{e}$ siècle (348-817), Villeneuve-d'Ascq, 1998 (Histoire et civilisations), p. 192.

30. La charte d'émancipation de Berthedrifus mentionne « la règle de saint Benoît et de saint Colomban " (L. Morelle, "Le statut d'un grand monastère... », p. 207). Sur la question de l'ordre monastique dans les fondations de la reine Bathilde, voir Alain Dierkens, « Prolégomènes à une histoire des relations culturelles entre les îles Britanniques et le continent pendant le haut Moyen Âge : la diffusion du monachisme dit colombanien ou iro-franc dans quelques monastères de la région parisienne au viI ${ }^{\mathrm{e}}$ siècle et la politique religieuse de la reine Bathilde », dans H. AtsmA (éd.), La Neustrie..., vol. II, p. 371-393.

31. Voir Michel Rouche, «L'héritage de la voirie antique dans la Gaule du haut Moyen Âge ( $\mathrm{v}^{\mathrm{e}}$-xi $\mathrm{x}^{\mathrm{e}}$ siècle) ", dans L'homme et la route en Europe occidentale au Moyen Âge et aux Temps modernes (Centre culturel de Flaran, $2^{\text {des }}$ Journées internationales d'histoire, 20-22 septembre 1980), Auch, 1982 (Flaran, 2), p. 13-32.

32. Voir Stéphane Lebeco, "La Neustrie et la mer », dans H. Atsma (éd.), La Neustrie..., vol. I, p. 405-440, en particulier p. 414 ; S. Lebeco, "Entre Antiquité tardive et très haut Moyen Âge : permanence et mutations des systèmes de communication dans la Gaule et ses marges ", dans ID., Hommes, mers et terres du Nord au début du Moyen Âge, Villeneuve-d'Ascq, 2011 (Histoire et civilisations), t. II, Centres, communications, échanges, p. 177-204 [1 ${ }^{\text {re }}$ éd. dans Morfologie sociali e culturali in Europa fra tarda antichità e alto medioevo ( Centro italiano di studi sull'alto medioevo, Spoleto, 3-9 aprile 1997), Spolète, 1998 (Settimane di studio del Centro italiano di studi sull'alto medioevo, 45), p.461-502], et iD., Bruno BÉthouART et Laurent VersLYPE (éd.), Quentovic, : environnement, archéologie, histoire (actes du colloque international de Montreuil-sur-Mer, Étaples et Le Touquet et de la journée d'études de Lille sur les origines de Montreuil-sur-Mer, 11-13 mai 2006 et $1^{\text {er }}$ décembre 2006), Villeneuve-d'Ascq, 2010. 


\section{Le récit de la passion et de l'invention des trois saints martyrs Fuscien, Victoric et Gentien : les premiers témoins manuscrits}

Le récit hagiographique vient compenser la carence d'informations biographiques dont sont coutumières les formules pour le moins laconiques du martyrologe du pseudo-Jérôme. Les deux martyrs amiénois y sont associés à Gentien, ignoré jusque-là par les martyrologistes. Ainsi, le couple formé par Fuscien et Victoric, à l'image probablement de celui qui était composé à Milan par Gervais et Protais, Nazaire et Celse, et reproduit en Gaule du Nord par Crépin et Crépinien, Rufin et Valère, se transforme en une combinaison ternaire faisant écho au groupe constitué par saint Denis et ses compagnons Rustique et Éleuthère. Par ailleurs, les premiers témoins du récit, copiés au tournant des viII ${ }^{\mathrm{e}}$ et $\mathrm{IX}^{\mathrm{e}}$ siècles et conservés respectivement à Paris (Bibl. nat. de France, Lat. 12598, fol. 32v-35v) et à Vienne (Österreichische Nationalbibliothek, Lat. 371, fol. 220v-223v et 11lr-112v) ${ }^{33}$, modifient la tradition martyrologique en attribuant la préséance à Fuscien sur Victoric.

L'exemplaire conservé à la Bibliothèque nationale de France, dont on est sûr qu'il appartenait à la bibliothèque de Corbie au moins depuis Maurdramne, comme l'attestent les corrections et les adjonctions transcrites dans l'écriture qui porte le nom de l'abbé, est un légendier composite, dont la structure et les différentes étapes de réalisation ont été restituées par Michèle Gaillard ${ }^{34}$. Les textes de la Passio sanctorum Fusciani, Victorici (fol. 32v35v) [BHL 3226] et de l'Inventio ipsorum corporum (fol. 35v-37v) [BHL 3229] font partie d'un ensemble qui intègre des Vies de saints de la province ecclésiastique de Reims (fol. 23r-46r). Les deux récits sont précédés des Vitae des trois grands évêques Remi, Médard et Vaast (fol. 23r-32r) et suivis des Passions des saints Just, Lucien, Crépin et Crépinien (fol. 37v-46r). À l'origine, cette partie était solidaire de celle, écrite par une autre main, qui est située à la fin du volume, qui comprend les Passions de saintes martyres et la Vie de saint Germain d'Auxerre (fol. 62r-107r) ${ }^{35}$. Entre les deux a été intercalée une section, un peu plus ancienne, consacrée aux Vies des saints Servais et Lambert (fol. 47r-6lr) qu'Elias A. Lowe a attribué à un atelier situé soit dans le Nord-Est de la France soit aux Pays-Bas, dans le voisinage d'Utrecht ou de Maastricht, eu égard à la vénération dévolue aux deux évêques dans ces régions ${ }^{36}$. L'éminent paléographe a également proposé de situer dans ces mêmes régions, sans toutefois mentionner Utrecht ou Maastricht, l'atelier

33. M. Gaillard, « Remarques sur les plus anciennes versions de la Passio et de l'Inventio des saints Fuscien, Victoric et Gentien (manuscrits Paris, BNF, Lat. 12598 et Wien, ÖNB, 371) ", dans Monique Goullet (éd.), Parva pro magnis munera : études de littérature tardo-antique et médiévale offertes à François Dolbeau par ses élèves, Turnhout, 2009 (Instrumenta patristica et mediaevalia, 51), p. 397-409, et M. GAILlARD, "Un "cycle" hagiographique du haut Moyen Âge...".

34. EAD., "Remarques sur les plus anciennes versions de la Passio... ».

35. Pour le contenu exhaustif du volume, voir ibid. À l'exception de saint Just, tous les saints et saintes dont la Vita est présente dans le légendier ont leur nom inscrit dans le manuscrit Lat. 12260 de la Bibl. nat. de France.

36. E. A. Lowe, Codices Latini Antiquiores..., t. V, nº 644a. En réalité, il aurait été plus pertinent d'évoquer le diocèse de Tongres-Maastricht-Liège. 
responsable de la copie des textes contenus dans la section incluant les deux récits relatifs aux trois martyrs amiénois ${ }^{37}$. Sur le fondement de critères paléographiques, l'auteur assigne à ce même atelier la réalisation du manuscrit Latin 13396 de la Bibliothèque nationale de France ${ }^{38}$.

L'argument fondé sur le caractère régional du culte des saints peut conduire à émettre une toute autre proposition pour cette section, où l'accent a été délibérément mis sur les figures de saint Vaast d'Arras et des trois martyrs amiénois. Les intitulés des textes qui leur sont consacrés tranchent en effet singulièrement dans l'ensemble du corpus par le traitement particulier réservé aux capitales mêlées d'onciales qui les composent. Cela induit logiquement à penser que le scribe travaillait dans un scriptorium situé dans un diocèse où le culte de ces saints était privilégié. À vrai dire, la manière dont le copiste a mis en valeur les deux textes se rapportant aux trois martyrs fait pencher la balance du côté du diocèse d'Amiens. Agencé sur trois lignes, dans un espace équivalent à six lignes de réglure, l'en-tête de la Passio possède une ampleur qu'on ne retrouve nulle part ailleurs dans le manuscrit. La date de la célébration liturgique de l'Inventio est transcrite en capitales et selon le même module que l'intitulé sans qu'aucun autre texte n'ait reçu cette distinction. Enfin, concluant l'ensemble des deux récits, la formule «Expli[cit] feliciter ", écrite en capitales et dans un format similaire aux intitulés, fait figure d'exception dans le volume ${ }^{39}$. Ce qui pourrait être perçu comme le résultat d'une fantaisie du copiste relève à l'évidence $d$ 'une intention visant à valoriser les trois saints amiénois.

Certes, le type d'écriture utilisé dans le corps du texte, une minuscule caroline précoce riche en ligatures, n'est pas suffisamment bien caractérisé pour déterminer avec certitude son lieu de production. Mais, à la différence d'Elias A. Lowe et de David Ganz ${ }^{40}$, Michèle Gaillard n'exclut pas une transcription dans la région de Corbie, voire à Corbie même ${ }^{41}$. Un indice pourrait confirmer cette hypothèse. On observe en effet dans cette partie du recueil une pratique d'ordre codicologique très fréquemment utilisée par les scribes de Corbie avant le milieu du $\mathrm{Ix}^{\mathrm{e}}$ siècle, qui consiste à disposer le premier feuillet de chaque cahier de telle sorte qu'il présente son côté chair à l'extérieur, alors que, dans la majorité des scriptoria de l'époque, l'habitude était de présenter le côté poil à l'extérieur ${ }^{42}$.

Un autre argument en faveur d'une origine corbéienne est le rapprochement proposé par Elias A. Lowe avec le manuscrit Latin 13338 de la Biblio-

37. Ibid., $\mathrm{n}^{\mathrm{o}} 644 \mathrm{~b}$.

38. Ibid., $\mathrm{n}^{\circ} 661$.

39. Il semblerait que les copistes de Corbie avaient l'habitude d'utiliser cette formule, que l'on rencontre également au fol. 12r du manuscrit Lat. 12260 de la Bibl. nat. de France.

40. D. Ganz, Corbie in the Carolingian Renaissance..., p. 129, classe sans justification l'ensemble du légendier parmi les manuscrits étrangers à Corbie.

41. M. Gaillard, "Remarques sur les plus anciennes versions de la Passio... », p. 402-403.

42. T. A. M. Bishop, "The Script of Corbie... », et Jean Vezin, "La réalisation matérielle des manuscrits latins pendant le haut Moyen Âge ", dans Albert Gruys et Johann Peter GumberT (éd.), Codicologica, t. II, Éléments pour une codicologie comparée, Leyde, 1978 (Litterae textuales), p. 15-51. C'est également le cas pour la section contenant les Vies des saints Servais et Lambert. 
thèque nationale de France. Marianne Besseyre a attiré l'attention sur la présence dans ce codex d'un motif caractéristique d'un grand nombre de productions liées à Corbie, consistant en un rond noir placé au centre d'un cercle blanc, qui rend crédible la probabilité que le volume, attesté à Corbie au Ix ${ }^{\mathrm{e}}$ siècle, ait été copié dans le scriptorium de l'abbaye ${ }^{43}$. Étant donné que cette marque de fabrique figure dans l'initiale qui introduit la Passion de sainte Cécile située au fol. 62r de notre légendier, nous sommes autorisés à en tirer une conclusion identique.

Le manuscrit de Vienne est un passionnaire dans lequel les martyrs sont classés selon l'ordre du calendrier liturgique. La Passio et l'Inventio sont, pour cette raison, dissociées à l'intérieur du volume. Celui-ci provient de la bibliothèque épiscopale de Salzbourg et fut copié, selon Bernhard Bischoff ${ }^{44}$, au monastère de Saint-Amand à l'époque où l'archevêque de Salzbourg, Arn (798-821), en était l'abbé.

Dans l'analyse comparative qu'elle a consacrée aux deux plus anciens témoins de la Passio et de l'Inventio, Michèle Gaillard a montré que leurs textes étaient très proches l'un de l'autre par leur contenu et leur transcription à peu de temps d'intervalle, avec cependant une antériorité pour le Latin 12598, dont la datation à l'extrême fin du viII ${ }^{\mathrm{e}}$ siècle est largement acceptée par la critique ${ }^{45}$. L'auteur en déduit l'hypothèse qu'ils pourraient dériver directement ou indirectement d'un modèle commun, aujourd'hui perdu, comme le laisse entendre l'incise « Nam, ut [hi] storiae gesta commemore [nt]", transcrite dans le récit de la Passio.

Le récit, tel qu'il nous est parvenu ${ }^{46}$, n'échappe pas aux anachronismes ni aux lieux communs hagiographiques, propres à la loi du genre. Mais il contient des informations qui pourraient nous mettre sur la piste de l'époque d'écriture de cet archétype. Le texte s'ouvre sur le groupe de douze missionnaires qui, sous la conduite de Denis, partirent de Rome pour évangéliser le Nord de la Gaule. Aux côtés de Fuscien et Victoric figurent Piat, Rufin, Valère, Crépin, Crépinien, Lucien, Marcel, Quentin et Rieul. Sept d'entre

43. Marianne Besseyre, notice du manuscrit Latin 13338, dans Marie-Pierre Laffitte et Charlotte DenoëL, Trésors carolingiens : livres manuscrits de Charlemagne à Charles le Chauve [expos. Paris, Bibliothèque nationale de France, 20 mars-24 juin 2007], Paris, 2007, nº 20, p. 126-127. Le motif apparaît dans les initiales des fol. 2r et 38v, mais également dans l'image de dédicace du fol. $1 \mathrm{v}$.

44. L'identification du scriptorium revient à Bernhard Bischoff, qui a classé le manuscrit parmi les témoins paléographiques du "style d'Arn» (B. Bischoff, Die südostdeutschen Schreibschulen und Bibliotheken in der Karolingerzeit, t. II, Die vorwiegend österreichischen Diözesen, Wiesbaden, 1980, p. 120-121).

45. Martin Heinzelmann, «L'hagiographie mérovingienne : panorama des documents potentiels », dans M. Goulet, M. Heinzelmann et Christiane Veyrard-Cosme (éd.), L'Hagiographie mérovingienne à travers ses réécritures, Ostfildern, 2010 (Beihefte der Francia, 71), p. 27-82, spéc. p. 73.

46. Une édition de la Passion [BHL 3226] et de l'Invention [BHL 3229] a été publiée, en même temps qu'une version plus récente de la Passion [BHL 3224], par Charles SALmon, «Actes inédits des saints martyrs Fuscien, Victoric et Gentien », dans Mémoires de la Société des Antiquaires de Picardie, t. 8 (1861), p. 113-154. Plus récemment, une réédition du texte de la Passion [BHL 3226] a été proposée par Ritva Jonsson, Historia : études sur la genèse des offices versifiés, Stockholm, 1968 (Acta universatis Stockholmiensis. Studia Latina Stockholmiensia, 15), p. 210213. 
eux, Fuscien et Victoric, Rufin et Valère, Crépin et Crépinien ainsi que Quentin, sont censés, d'après leur Passion, avoir été persécutés, sous le règne conjoint de Dioclétien et Maximien (286-305), par un préfet dénommé Riciovar dont l'historicité est unanimement rejetée aujourd'hui. D'autres, tels Lucien et Piat, ne sont pas présentés dans les textes comme des victimes de Riciovar, mais sont associés dans la tradition hagiographique à Quentin, Crépin et Crépinien, Fuscien et Victoric ${ }^{47}$. Sur la foi du témoignage des écrits de Grégoire de Tours, composés entre 573 et $594{ }^{48}$, de la recension gallicane du martyrologe hiéronymien mise en chantier aux alentours de 600 49, et de la Vita Eligii [BHL 2474], rédigée par saint Ouen dans le courant des années $670^{50}$, le culte de ces martyrs, compagnons de Fuscien et Victoric, était en place dès l'époque mérovingienne, soit bien avant que le rédacteur de la Passio sanctorum Fusciani et Victorici ait pris la plume.

La probabilité que le culte des martyrs censés avoir évangélisé le Nord de la Gaule ait pu générer très tôt des récits est avérée, en particulier par les recherches menées autour de saint Quentin. Il a été démontré que la Passio Quintini [BHL 6999-7000] avait été écrite dès la fin $\mathrm{du} \mathrm{vI}^{\mathrm{e}}$ ou le début du viI ${ }^{\mathrm{e}}$ siècle, avant même qu'Éloi, évêque de Noyon et de Tournai (640-660), n'ait pris la décision d'agrandir le sanctuaire, pour des raisons éminemment politiques ${ }^{51}$. Or, dans la Passion de Fuscien et Victoric, la référence à Quentin est explicite : après avoir prêché à Thérouanne, les deux missionnaires se rendent à Amiens dans l'espoir d'y retrouver Quentin. Sur le chemin de Paris, ils rencontrent Gentien, non encore converti à la foi chrétienne, qui leur apprend le martyre de leur ancien compagnon de voyage par Riciovar à

47. L. Duchesne, Fastes épiscopaux de l'ancienne Gaule, t. III, Les provinces du Nord et de l'Est, Paris, 1915, p 141-152 ; Brigitte Merjns et Charles Mériaux, « Le cycle de Rictiovar et la topographie chrétienne des campagnes septentrionales à l'époque mérovingienne » dans Dominique Paris-Poulain, Sara Nardi-Combescure et Daniel Istria (éd.), Les premiers temps chrétiens dans le territoire de la France actuelle : hagiographie, épigraphie et archéologie, nouvelles approches et perspectives de recherche (actes du colloque international d'Amiens, université de Picardie Jules-Verne, faculté des arts, 18-20 janvier 2007), Rennes, 2009 (Archéologie et culture), p. 19-33, et M. GaIllard, "Remarques sur les plus anciennes versions de la Passio... ».

48. Grégoire de Tours cite Quentin (Liber in gloria martyrum, éd. B. KRusaH, MGH, SS rer. Merov., t. I, part. II, rééd. Hanovre, 1969, chap. LxxiI, p. 86-87), Marcel (Libri historiarum X, éd. B. Krusch et Wilhelm Levison, MGH, SS rer. Merov., t. I, part. I, 2 e éd., Hanovre, 1937-1951, liv. IX, chap. III, p. 415-416 ; liv. IX, chap. XXviI, p. 445-446, et liv. X, chap. x, p. 494, et Liber in gloria martyrum..., chap. LII, p. 75), et mentionne la basilique des saints Crépin et Crépinien (Libri historiarum X..., liv. V, chap. xxxıv, p. 238-241, et liv. IX, chap. Ix, p. 421-424).

49. On notera cependant l'inscription tardive dans les codices pleniores de Piat et Rieul. Dans la version abrégée de la recension gallicane du manuscrit Lat. 12260 de la Bibl. nat. de France manquent les noms de ces deux martyrs ainsi que ceux de Rufin et Valère.

50. Le texte fait état de la découverte par l’évêque de Noyon et de Tournai des restes de Quentin, Piat et Lucien, et mentionne la translation des corps de Crépin et Crépinien (voir C. Mériaux, "Du nouveau sur la Vie de saint Éloi », dans Mélanges de science religieuse, t. 67 (2010), p. 71-85.

51. Voir M. Gaillard et Jean-Luc Villette, "L'histoire de saint Quentin entre textes et images ", dans Hervé Cabezas (dir.), Aux origines de Saint-Quentin : de la tradition littéraire à la réalité archéologique [expos. Saint-Quentin, musée Antoine-Lécuyer, 16 nov. 2011-13 févr. 2012], Saint-Quentin, 2011, p. 7-14, et M. GaIllard et Christian SAPIN, «Autour de la tombe de saint Quentin : histoire et archéologie d'un culte (milieu Iv ${ }^{\mathrm{e}}$-début viI ${ }^{\mathrm{e}} \mathrm{s}$.) », dans M. GAILLARD (éd.), L'empreinte chrétienne en Gaule du IV au IX siècle, Turnhout, 2014 (Culture et société médiévales, 26), p. 271-288. 
Augusta Viromandorum 52. C' est en essayant vainement de rejoindre Quentin qu'ils vont au-devant du martyre. La part accordée à Quentin dans le récit suggère une dépendance littéraire qui conduit à dater la rédaction du texte primitif dans le courant du viI siècle. En comparant les Passions des missionnaires actifs dans le Nord de la Gaule (Fuscien et Victoric, Crépin et Crépinien, Lucien, Quentin, Piat), Michèle Gaillard avance l'hypothèse d'une rédaction de la Passion des deux martyrs amiénois à la fin de ce siècle ${ }^{53}$. Rien ne prouve cependant que le texte parvenu jusqu'à nous soit conforme à la version primitive.

Les incohérences chronologiques du récit, qui n'avaient pas échappé à Louis Duchesne ${ }^{54}$, révèlent les intentions du rédacteur. Maximien n'appliqua pas en Gaule les édits de persécutions édictées par Dioclétien, mais présenter ces saints comme ayant subi le martyre à l'époque de la grande persécution de Dioclétien les auréolait d'un incontestable prestige et contribuait à légitimer leur culte. Denis n'a pu, au début du Iv siècle, accompagner des missionnaires venus de Rome, mais le placer à leur tête conférait à ces derniers l'apostolicité dont il avait été investi par le pape Clément, successeur de saint Pierre. En témoigne leur nombre, clairement établi sur le modèle apostolique, en totale contradiction avec le récit de Grégoire de Tours, qui présente Denis comme faisant partie d'un groupe de sept prédicateurs envoyés en Gaule sous l'empereur Dèce (249-251) ${ }^{55}$.

Le chiffre trois, éminemment symbolique, associé aux martyrs amiénois est un autre élément signifiant, qui fait écho à Denis et ses compagnons, dont la première Passio, dite gloriosae [BHL 2171], rédigée très probablement à la fin $\mathrm{du} \mathrm{vi}^{\mathrm{e}}$ siècle ${ }^{56}$, affirme expressément que leur triple martyre glorifie l'indivisible trinité de Dieu. Un exemplaire de cette version fut copié à Corbie en minuscule caroline de «type Maurdramne » 57 , donnant à penser, ainsi que l'a suggéré Jean Vezin, que les scriptoria de Saint-Denis et de Corbie entretenaient d'étroites relations ${ }^{58}$.

52. Le nom exact est Augusta Vermanduorum.

53. M. Gaillard, "Remarques sur les plus anciennes versions de la Passio... ».

54. L. Duchesne, Fastes épiscopaux..., t. III, p. 147.

55. Grégolre de Tours, Libri historiarum X..., liv. I, chap. xxx, p. 22-23. Un exemplaire, copié à la fin du viI è siècle, était conservé à Corbie (auj. Bibl. nat. de France, Lat. 17655).

56. Voir en dernier lieu M. Heinzelmann, "La passion Gloriosae de saint Denis (BHL 2171) ", dans Bibliothèque de l'École des chartes, t. 172 (2014), p. 13-28.

57. Saint-Pétersbourg, Bibl. nat. de Russie, Lat. F. v. I. 12 (nº IV), fol. 63r-68r. Voir E. A. Lowe, Codices Latini Antiquiores..., t. XI, Hungary, Luxembourg, Poland, Russia, Spain, Sweden, The United States and Yugoslavia, Oxford, 1966, n 1609.

58. J. VEzIN, «Les relations entre Saint-Denis et d'autres scriptoria pendant le haut Moyen Âge ", dans Peter Ganz (éd.), The Role of the Book in Medieval Culture : Proceeding of the Oxford International Symposium, 26 September-1 October 1982, vol. I, Turnhout, 1986 (Bibliologia : elementa ad librorum studia pertinentia, 3), p. 17-39, spéc. 20. La présence dans deux manuscrits copiés à Corbie (Bibl. nat. de France, Lat. 13025 et 14087) d'une écriture caractéristique de Saint-Denis, du type de l'abbé Fardulphe (797-806), atteste la réalité des contacts entre les copistes des deux abbayes (J. VezIN, "Les manuscrits copiés à Saint-Denis en France pendant l'époque carolingienne ", dans Paris et Île-de-France : mémoires publiés par la Fédération des sociétés historiques et archéologiques de Paris et l'Île-de-France, t. 32 (1981) [dossier « Le Moyen Âge en Île-de-France »], p. 273-287. 
La Passio Fusciani et Victorici s'achève avec le miracle de la céphalophorie par lequel Fuscien et Victoric expriment leur souhait de reposer pour l'éternité auprès de Gentien. Selon le récit, ce dernier avait eu la tête tranchée pour s'être interposé entre Riciovar et les deux prédicateurs à qui il avait offert l'hospitalité. Après avoir été arrêtés, enchaînés et avoir enduré les supplices les plus cruels sur la route les conduisant à Amiens, Fuscien et Victoric connaissent le même sort. C' est alors qu'ils se redressent, se saisissent de leur tête et rebroussent chemin vers la demeure de Gentien, distante à plus d'un mille, afin de reposer auprès de celui qui « devait partager leur gloire dans les cieux ». Devenu un topos de la littérature hagiographique, cet épisode se rencontre également dans la deuxième version remaniée de la Passio Dionysii, dite Post beatam et gloriosam [BHL 2178], qui serait antérieure, selon Anne-Marie Helvétius, aux années $780{ }^{59}$. Cette version qui met en scène saint Denis portant sa tête dans ses bras et marchant sur une distance d'environ deux milles a pu fournir un modèle à l'auteur de la Passio sanctorum Fusciani et Victorici ${ }^{60}$.

Si la présence d'éléments narratifs identiques dans les Passions des martyrs ou les Vies de saints donne à penser qu'elles ont pu s'influencer mutuellement, la question de leur dépendance reste difficile à prouver. Ainsi, la prédication de Fuscien et Victoric à Thérouanne dont fait état leur Passion invite à s'interroger sur les liens éventuels de ce texte avec le Libellus miraculorum Bertini [BHL 1291], composé plus tardivement, entre 891 et 900, et qui mentionne très précisément à proximité de cette cité un village nommé Locus ecclesiae, parce que, dit-on, s'y trouvait une église que Fuscien et Victoric avaient construite «lors des prémices du christianisme parmi cette population " ${ }^{61}$. Or il n'est nullement question de cette église dans leur Passion. L'auteur des Miracles de saint Bertin l'a-t-il extrapolée du récit de la Passion? Avait-il à sa disposition une autre source écrite? Ou s'est-il conformé à une tradition orale, ainsi que le laisse entendre la formulation utilisée pour signaler cette église dans sa relation du raid normand sur le monastère de Sithiu en 891 ?

Si l'on accorde crédit au texte de l'Inventio ipsorum corporum, c'est sous l'épiscopat d'Honoré, à l'époque où le roi franc Childebert $I^{\text {er }}$ résidait à Paris (511-558), que furent découverts les corps saints. Le lieu d'ensevelissement est miraculeusement révélé par un ange à un prêtre d'Amiens, dénommé Lupicin, dont le chant de louange entonné à la découverte des reliques parvient, à cinq milles de là, aux oreilles de l'évêque d'Amiens, Honoré. Ayant appris la nouvelle, le roi des Francs Childebert envoie des émissaires afin qu'ils puissent transporter les corps jusqu'à Paris. Mais, à la suite d'un prodige, la translation échoua: les corps devinrent si pesants qu'il était

59. Voir en dernier lieu Anne-Marie Helvétius, « La deuxième version latine de la Passion de saint Denis (BHL 2178) ", dans Bibliothèque de l'École des chartes, t. 172 (2014), p. 29-60.

60. Pousser plus loin l'enquête sur la genèse et la tradition textuelle de la céphalophorie ambulatoire dépasserait le cadre de cette étude.

61. C. Mériaux, Gallia irradiata : saints et sanctuaires dans le Nord de la Gaule du haut Moyen Age, Stuttgart, 2006 (Beiträge zur Hagiographie, 4), p. 172. 
impossible de les déplacer. Childebert ordonna alors de les ensevelir et de construire en cet endroit un sanctuaire où les fidèles viendraient les vénérer et où s'accomplirent rapidement de nombreux miracles. L'incertitude plane sur l'historicité d'Honoré, nommé pour la première fois dans cette source. Quant à Childebert $\mathrm{I}^{\mathrm{er}}$, son implication dans la translation ne repose que sur la foi que l'on prêtera ou non à la capacité du texte à rendre compte de la réalité des faits ${ }^{62}$. Associer le roi franc à la découverte des reliques des trois martyrs permettait de revendiquer une ancienneté qui leur faisait probablement défaut. Recourir au miracle d'une translation interrompue contribuait à l'authentification d'un lieu que la tradition associait à la sépulture de saints martyrs.

Plusieurs motivations ont pu présider à l'écriture du récit hagiographique : celle d'offrir par exemple une identité plus tangible à deux supposés martyrs dont seuls les noms et le lieu de supplice étaient parvenus à la connaissance de la communauté chrétienne par l'intermédiaire du martyrologe hiéronymien, ou encore celle de promouvoir un culte local autour d'une sépulture, et consolider de facto l'autorité de l'évêque d'Amiens. Comme le pensait Louis Duchesne, l'activité d'Éloi visant à promouvoir des lieux de culte a pu se répercuter dans la promotion des cultes locaux des diocèses limitrophes. L'invention des saints Lucien et Piat, la translation des saints Crépin et Crépinien et l'élévation du corps de saint Quentin que lui crédite sa Vita, ne sont certainement pas restées sans effet auprès d'un évêque à la tête d'un diocèse qui manquait cruellement de loca sanctorum.

Il était sans aucun doute relativement aisé, pour un évêque en quête de reliques, de prélever dans une ancienne nécropole les sacro-saints ossements nécessaires à l'établissement d'un culte. Or, comme l'ont révélé les fouilles menées par l'abbé Messio entre 1863 et 1874 63, une nécropole d'époque gallo-romaine et mérovingienne existait bien sur le site où s'élève aujourd'hui l'église de Sains-en-Amiénois. Un autre scénario, différent de celui qui est proposé par les Actes des trois martyrs et lié à la christianisation des campagnes environnant la cité épiscopale, pourrait bien ainsi être avancé.

\section{Le développement du culte des trois martyrs au $\mathrm{Ix}^{\mathrm{e}}$ siècle}

Au vu des sources dont nous disposons, il ne semble pas que le culte des trois martyrs ait eu une large diffusion avant le $\mathrm{Ix}^{\mathrm{e}}$ siècle. Au contraire de plusieurs saints, cités dans la Passio Fusciani et Victorici, tels Quentin,

62. Sur la responsabilité de Childebert dans la christianisation du paysage gaulois, voir Bruno DumézIL, "La royauté franque et la christianisation des Gaules : le "moment" Childebert I (511-558) ", dans D. Paris-Poulain, S. Nardi-Combescure et D. Istria (éd.), Les premiers temps chrétiens..., p.41-49. Il n’est pas sans intérêt de noter la présence à Corbie au viII ${ }^{\mathrm{e}}$ siècle du praeceptum de Childebert qui visait à éradiquer les pratiques du paganisme. Le texte, copié au $\mathrm{VI}^{\mathrm{e}}$ ou $\mathrm{VII}^{\mathrm{e}}$ siècle, est intégré dans la «collectio Corbiensis", qui rassemble une collection canonique composite transmise par le manuscrit Latin 12097 de la Bibl. nat. de France.

63. Adolphe Messio, Sains et ses martyrs : Actes des saints Fuscien, Victoric et Gentien, Amiens, 1869. 
Crépin et Crépinien, Rufin et Valère, Lucien, Piat, associés à Denis, Rustique et Eleuthère, les noms des trois martyrs amiénois ne figurent pas dans les litanies, copiées entre 795 et l'an 800, dans le scriptorium de l'abbaye NotreDame de Soissons ${ }^{64}$, ni dans celles de la même époque du psautier dit de Charlemagne ${ }^{65}$, que celui-ci, alors roi des Francs, offrit à Angilbert pour l'abbaye Centula/Saint-Riquier. Ils sont en revanche bien mentionnés dans deux des trois listes de litanies transcrites peu après l'an 800 dans le psautier dit de Corbie de la bibliothèque d'Amiens ${ }^{66}$, et dans celles qui sont datées de la seconde moitié du $\mathrm{Ix}^{\mathrm{e}}$ siècle et qui proviennent d'un autre psautier, également d'origine corbéienne, conservé à Zürich ${ }^{67}$.

Le même constat peut être observé au sujet de la diffusion de leurs reliques. Elles sont absentes du lot recueilli au cours de la dernière décennie du VIII ${ }^{\mathrm{e}}$ siècle par Angilbert (794-814) pour la consécration de l'abbatiale de Centula/Saint-Riquier, alors que l'on dénombre, parmi d'autres, celles de Quentin, Crépin, Crépinien, Denis, Rustique et Éleuthère ${ }^{68}$. Cette lacune fut comblée, ainsi que nous l'apprend Hariulf, par l'évêque Hilmeradus (849871) lorsque, à la demande du gardien de l'abbaye, il consentit à lui accorder, entre juin 855 et la fin de l'année 865, quelques os des genoux des trois martyrs ${ }^{69}$. Puis ce fut au tour de l'évêque Otgarius (892/893-928) de concéder, en 893, à l'abbaye de Corbie, une partie du corps de Gentien, et à l'abbatiale, future collégiale, de Saint-Quentin, des reliques corporelles de Victoric ${ }^{70}$.

Pendant la même période, plusieurs recensions des martyrologes historiques qui incorporent des éléments narratifs du récit hagiographique contribuèrent à consolider les assises de la vénération dont les trois martyrs furent l'objet. Je me contenterai de citer le plus ancien exemplaire connu du martyrologe de Raban Maur, copié entre 844 et 854 71, qui introduit pour la première fois la commémoration de l'invention des corps de nos martyrs le 5 des calendes de juillet.

64. Montpellier, bibl. interuniv., section Médecine, H 409, fol. 34lv-342r.

65. Bibl. nat. de France, Lat. 13159, fol. 164v-165v.

66. Amiens, bibl. mun., ms. 18, fol. 142r et 143r. Ces litanies sont d'une autre main que celle qui a copié les psaumes et les cantiques du codex. La première liste s'achèvant par une invocation à l'empereur (fol. 141r), les litanies sont postérieures à l'an 800. Voir Astrid KRÜGER, LitaneiHandschriften der Karolingerzeit, Hanovre, 2007 (Monumenta Germaniae historica. Hilfsmittel, 24), p. 125-129, 600-601 et 606.

67. Zürich, Zentralbibliothek, Ms. Car. C.161, fol. 180r. Voir Astrid KRÜGER, LitaneiHandschriften der Karolingerzeit..., p. 193-194.

68. Hariulf, Chronique de l'abbaye de Saint-Riquier ( $V^{e}$ siècle-1104), éd. Ferdinand Lot, Paris, 1894 (Coll. de textes pour servir à l'étude et à l'enseignement de l'histoire, 17), liv. II, chap. Ix, p. 65.

69. Ibid., liv. III, chap. xII, p. 122.

70. Le récit de la translation de Gentien à Corbie nous est parvenu sur deux feuillets écrits au $\mathrm{XI}^{\mathrm{e}}$ siècle qui ont été ajoutés au sacramentaire dit de Corbie copié après 853 (Bibl. nat. de France, Lat. 12051, fol. lv-2r). Voir également, non seulement pour Gentien, mais aussi pour Victoric, Translatio reliquiarum sancti Gentiani martyris in monasterium Corbeiense, dans Acta sanctorum ordinis Sancti Benedicti, éd. Luc D’Achery et Jean Mabillon, t. IV, part. II, Paris, 1680, p. 486-489. Le chanoine Nicolas de Lestocq nous apprend que l'évêque avait déjà donné auparavant des parcelles de plusieurs saints dont celles des trois martyrs amiénois (Nicolas DE LEsTOco, Dissertation sur la translation du corps de s. Firmin le Confesseur, troisieme eveque d'Amiens [...], Amiens, 1711, p. 51).

71. Saint-Gall, Stiftsbibliothek, Cod. Sang. 457, fol. 83r. 
Selon de fortes probabilités, les responsables de l'abbaye de Corbie, avec l'assentiment de l'autorité épiscopale, ont eu une part active dans l'essor et le développement du culte des trois martyrs. Un témoignage plus tardif de la profonde dévotion des moines envers ces derniers le montre de manière significative. Il s'agit du très bref calendrier qui précède une première version des statuts d'Adalhard, copiée après 987, sous l'abbatiat de Maingaudus ${ }^{72}$ : à la suite des fêtes à caractère universel célébrant la Vierge, les apôtres, les saints Étienne, Laurent, Martin et Grégoire, prennent place des fêtes propres à l'abbaye comme celles de Bathilde et Theodefridus, puis un nombre très sélectif de célébrations se rapportant aux saints du diocèse, nommément Firmin, Fuscien, Victoric et Gentien.

\section{Nouvelles perspectives de recherche}

À la lumière des sources manuscrites examinées dans cet essai, il semblerait bien que l'abbaye de Corbie ne fût pas totalement étrangère à la promotion d'un culte circonscrit à la cité épiscopale et à ses alentours. Réservé dans un premier temps aux seuls Victoric et Fuscien, ce culte est attesté à l'époque où s'élaborent différentes compilations de la version gallicane du martyrologe. Plus tardivement, vers la fin du viI ${ }^{\mathrm{e}}$ siècle, semble-t-il, l'écriture d'une Vita qui associait aux deux martyrs amiénois un disciple nommé Gentien contribua à assurer une assise à la dévotion de ces saints.

De manière tout à fait hypothétique, l'émergence du culte des deux premiers martyrs pourrait alors être fixée dans une période fixée entre la réalisation à Corbie d'une compilation de la version auxerroise du martyrologe hiéronymien enrichie de leurs noms et l'utilisation de cette version comme modèle par le copiste du martyrologe d'Echternach, soit approximativement entre les années 670 et la première décennie de l'an 700. Cette fourchette ne fournissant qu'un terminus ante quem, il n'est pas interdit de penser que la genèse de ce culte ait pu éclore en amont de cette période autour d'une sépulture privilégiée, ce qui implique la prise de compte des données archéologiques.

Les fouilles effectuées par l'abbé Messio ont permis de mettre au jour le sarcophage dont l'existence était connue depuis les investigations de 1663 et différentes structures appartenant à d'anciennes constructions ${ }^{73}$. Malheureusement, ces fouilles mal documentées ne suffisent pas à nous éclairer. Soucieux de mettre en accord ses découvertes avec le récit hagiographique,

72. Bibl. nat. de France, Lat. 13908, fol. 1v-2r. Voir L. Morelle, « La liste des repas commémoratifs offerts aux moines de l'abbaye de Corbie (vers 986/989) : une nouvelle pièce au dossier du "Patrimoine de saint Adalhard" ? ", dans Revue belge de philologie et d'histoire, t. 69 (1991), p. 279-299.

73. A. Messio, Sains et ses martyrs ... Voir la synthèse de ces fouilles dans Florence FuUtre et Bernard Mrlle, Prospection systématique pédestre sur la commune de Sains-en-Amiénois, Service régional de l'archéologie de Picardie, 1992, rapport conservé à la direction régionale des Affaires culturelles de la région Hauts-de-France. 
l'abbé n'hésita pas à dater la sépulture du temps où les trois martyrs auraient été persécutés. Sur le fondement des données apportées par les textes, il aurait été plus logique de situer la réalisation du sarcophage peu après la découverte des corps saints, d'autant plus que l'abbé a cru reconnaître dans les structures qu'il avait dégagées le sanctuaire censé avoir été bâti sur l'ordre de Childebert $\mathrm{I}^{\mathrm{er}}$.

Un nouvel éclairage sur l'émergence du culte des martyrs amiénois et les étapes de son développement pourrait être apporté à l'issue d'une nouvelle campagne de fouilles conduites avec les méthodes scientifiques requises.

Dominique Paris-Poulain, université de Picardie Jules-Verne, Textes, représentations, archéologie, autorité et mémoires de l'Antiquité à la Renaissance. 